\title{
A rare initial presentation of miliary tuberculosis
}

\author{
Somya Ish', Vidushi Rathi', Pranav Ish' , Himanshu Garkoti', Deepa Sharma' \\ 'Department of Ophthalmology, Dr Ram Manohar Lohia Hospital, New Delhi, India \\ ${ }^{2}$ Department of Pulmonary, Critical Care And Sleep Medicine, Vardhaman Mahavir Medical College \& Safdarjung Hospital, \\ New Delhi, India
}

A sixteen-year-old female presented with a gradual decrease of vision in the left eye for two weeks. She did not report any fever, weight loss, discharge, or redness of the eye. The chest X-ray of the patient was normal. On ocular examination, the best corrected visual acuity in the right eye was 6/6. In the left eye, the best acuity was noted when fingers were counted close to the face. Anterior segment examination of both the eyes was normal with normally reacting pupils. The fundus examination of the right eye was normal. In the left eye, a well-defined, subretinal, elevated, and oval-shaped lesion was located on the macula. It had a 1.5 $\mathrm{mm}$ disc diameter (DD) that had overlying dilated vessels and adjacent oedema with subretinal haemorrhage which was suggestive of a possible tuberculoma (Figure 1A). Optical coherence tomography (OCT) of the macula of the left eye revealed elevation of all the retinal layers along with parafoveal subretinal fluid and one intraretinal cystic space (Figure 1B). There was a history of pulmonary tuberculosis two years ago which was microbiologically diagnosed and adequately treated for six months.

The patient subsequently developed malaise, weight loss, and a positive tuberculin skin test. A repeat chest X-ray done 1 week after the initial presentation was suggestive of miliary tuberculosis (Figure 1C). There were no neurological deficits or symptoms of higher mental dysfunction. A non-contrast CT of the head was performed and the results were normal. A sputum sample was examined using the cartridge-based nucleic acid amplification test (CBNAAT) and returned positive for mycobacterium tuberculosis with no rifampicin resistance. The patient was treated with antitubercular therapy (ATT) alongside systemic steroids and showed symptomatic improvement.

Ocular TB is a rare presentation (1\% of all cases of TB) and can involve any part of the eye [1]. The most common intraocular manifestations of tubercular posterior uveitis include multiple choroidal tubercles and, less commonly, as a large solitary tuberculoma located at the posterior pole [2], which was seen in our case. Moreover, a solitary tuberculoma is often seen in the chronic course of the disease and, uncommonly, as the initial manifestation of miliary tuberculosis. Even though the exact mechanism remains unclear, the choroid
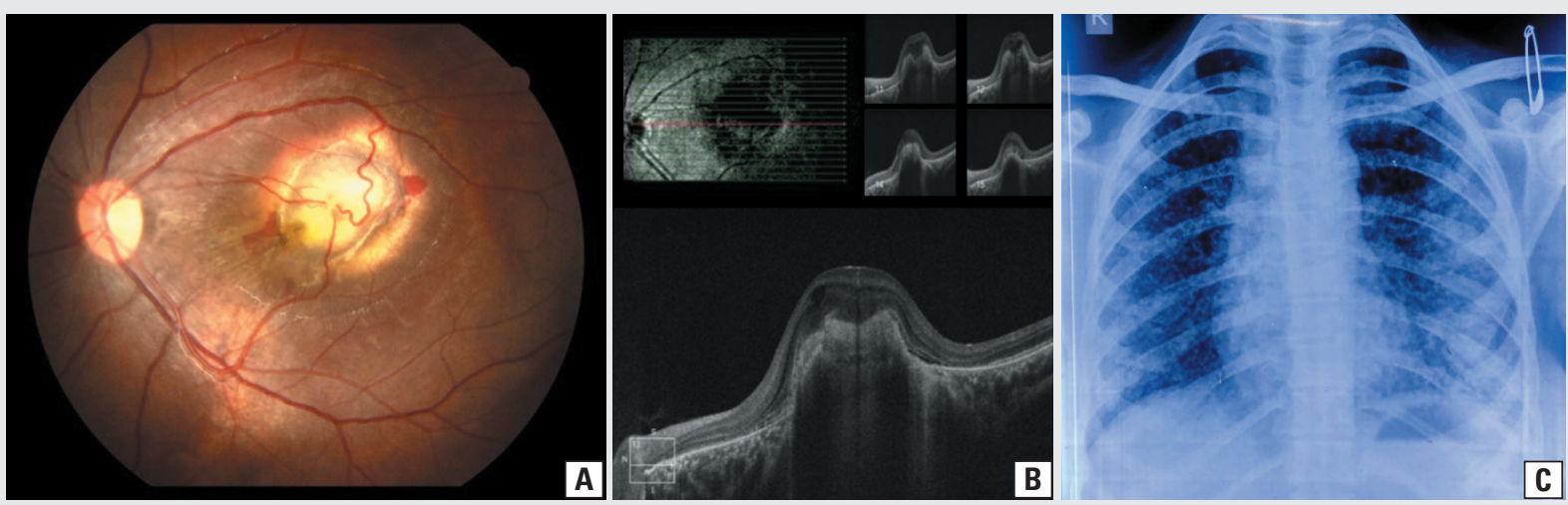

Figure 1. A. Fundoscopic examination of the left eye revealing a well-defined, subretinal, elevated, and oval-shaped lesion that was located on the macula. It had a $1.5 \mathrm{~mm}$ disc diameter (DD) that had overlying dilated vessels and adjacent oedema with subretinal haemorrhage, which was suggestive of a possible tuberculoma; B. Optical coherence tomography (OCT) of the macula of the left eye demonstrating an elevation of all the layers of the retina with parafoveal subretinal fluid and one intraretinal cystic space; C. Chest X-ray posterior-anterior view showing miliary shadows bilaterally suggestive of miliary tuberculosis

Address for correspondence: Pranav Ish, Department of Pulmonary, Critical Care And Sleep Medicine, Vardhaman Mahavir Medical College \& Safdarjung Hospital, New Delhi, India; e-mail: pranavish2512@gmail.com

DOI: 10.5603/ARM.a2020.0127

Received: 22.01.2020

Copyright (C) 2020 PTChP

ISSN 2451-4934

Conflict of interest: None 
tubercles may suggest direct hematogenous infection, whereas the vasculitis and choroiditis are more likely to be the result of immune hypersensitivity [3]. According to previously reported cases, affected patients often have healed pulmonary tuberculosis, although rarely choroidal manifestations may lead to the subsequent diagnosis of pulmonary tuberculosis [4]. This is what occurred in our case. Choroidal tuberculoma has also been reported as an isolated presentation with no extra-ocular tuberculosis [5].

This case highlights the truly systemic nature of disseminated tuberculosis. A high index of suspicion by doctors of various specialities can help in making an early diagnosis with an appropriate treatment plan to prevent morbidity.

\section{References:}

1. Bodaghi B, LeHoang P. Ocular tuberculosis. Curr Opin Ophthalmol. 2000; 11(6): 443-448, doi: 10.1097/00055735-20001200000010, indexed in Pubmed: 11141639.

2. Arej N, Fadlallah A, Chelala E. Choroidal tuberculoma as a presenting sign of tuberculosis. Int Med Case Rep J. 2016; 9: 365-368, doi: $10.2147 /$ IMCRJ.S119703, indexed in Pubmed: 27956845.

3. Shakarchi FI. Ocular tuberculosis: current perspectives. Clin Ophthalmol. 2015; 9: 2223-2227, doi: 10.2147/OPTH.S65254, indexed in Pubmed: 26648690.

4. Jabbour NM, Faris B, Trempe CL. A case of pulmonary tuberculosis presenting with a choroidal tuberculoma. Ophthalmology. 1985; 92(6): 834-837, doi: 10.1016/s0161-6420(85)33952-0, indexed in Pubmed: 4034179.

5. Mansour A, Haymond R. Choroidal tuberculomas without evidence of extraocular tuberculosis. Graefe's Archive for Clinical and Experimental Ophthalmology. 1990; 228(4): 382-383, doi: 10.1007/bf00920066. 